\title{
Implementation of Wireless Communication using Adaptive Beamforming of Smart Antenna
}

\author{
Manju Jain, Prof. Bhagwan Swaroop Sharma \\ M. Tech in microwave Dept. of ECE, RKDF, RGTU, Bhopal, M.P. India \\ Assistant professor, Dept. of ECE, RKDF, RGTU, Bhopal, M.P. India
}

\begin{abstract}
As the growing demand for mobile communications is constantly increasing, the need for better coverage, improved capacity, and higher transmission quality rises. Thus, a more efficient use of the radio spectrum is required. A smart antenna system is capable of efficiently utilizing the radio spectrum and is an effective solution to the present wireless system problems while achieving reliable and robust high-speed, high-data-rate transmission. Smart antenna technology offer significantly improved solution to reduce interference level and improve system capacity. Smart antenna technology attempts to address this problem via advanced signal processing technology called beamforming. Adaptive beamforming is used for enhancing a desired signal while suppressing noise and interference at the output of an array of sensors., in this paper work, a robust adaptive beamforming algorithm using smart antenna base station system, is to investigated its performance in presence of multipath components and multiple users. The capability of smart antenna and adaptive antenna easily employable to cognitive radio and OFDM system.
\end{abstract}

Keywords - Smart/adaptive antenna, beamforming, DSP, OFDM

\section{INTRODUCTION}

Over the last few years the demand for service provision via the wireless communication bearer has risen beyond all expectations. At the end of the last century more than 20 million users in the United States only utilized this technology [2]. At present the number of cellular users is growing annually by approximately 50 percent in North America, 60 percent in Western Europe, 70 percent in Australia and Asia and more than 200 percent in South America. The proliferation of wireless networks and an increase in the bandwidth required has led to shortages in the scarcest resource of all, the finite number of radio frequencies that these devices use. Generally, increased transmission rates require increased power and bandwidth independently of medium.

For a given amount of power (constrained by regulation or practical considerations) and a fixed amount of bandwidth (the amount one can afford to buy) there is a finite (small) amount of capacity (bits/sec/Hz/unit-area, really per unit-volume) that operators can sell to their customers, and a limited range over which customers can be served from any given location. Thus, the two basic problems that arise in such systems are:

A. How to acquire more capacity so that a larger number of customers can be served at lower costs maintaining the quality at the same time, in areas where demand is large (spectral efficiency)?

B. How to obtain greater coverage areas so as to reduce infrastructure and maintenance costs in areas where demand is relatively small (coverage)? This concern has led to the deployment of smart antenna systems throughout major metropolitan cellular markets. These smart antenna systems have typically employed multibeam technologies, which have been shown, through extensive analysis, simulation, and experimentation, to provide substantial performance Improvements in FDMA, TDMA and CDMA networks [3-7]. Multi beam architectures for FDMA and TDMA systems provide the straight-forward ability of the smart antenna to be implemented as a non-invasive add-on or appliqué to an existing cell site, without major modifications or special interfaces [8]. This paper mainly concentrate on use of smart antennas in mobile communications that enhances the capabilities of the mobile and cellular system such as faster bit rate, multi use interference, Space Division Multiplexing (SDMA), adaptive SDMA [9], increase in range, multipath mitigation, reduction of errors due to multipath Fading, best suitability of multi-carrier modulations such as OFDMA. The best application of smart antennas is its suitability for demand based frequency allocation in hierarchical system approach (flexible antenna pattern are achieved electronically and no physical movement of receiving antennas is necessary). The advantage of smart antennas application in cellular systems is decreased inter symbol interference, decreased cochannel Interference and adjacent channel interference, improved bit error rate (due to decreased amount of 
multipath and ISI), increase in receiver sensitivity, reduction in power consumption \& RF pollution. Smart antennas are most appropriate for use of cognitive radio (software radio technology provides flexibility) and the greatest advantage of smart antenna is a very high security. The main impediments to high-performance wireless communications are interference from other users (co channel interference), the Inter- Symbol Interference (ISI) and signal fading caused by multipath. Co-channel interference limits the system capacity, defined as the number of users which can be serviced by the system. However, since the desired signal and co-channel interference typically arrive at the receiver from different directions, smart antennas can exploit these differences to reduce co-channel interference, thereby increasing system capacity. The reflected multipath components of the transmitted signal also arrive at the receiver from different directions, and spatial processing can use these differences to attenuate the multipath, thereby reducing ISI and fading and results in improve reception by counteracting with negative effects of multipath fading by Adaptive Beamforming technique. This technique achieve maximum reception in a specified direction by estimating the signal arrival from a desired direction (in the presence of noise) while signals of the same frequency from other directions are rejected. There are several Adaptive beamforming algorithms as SMI, RLS, CMA varying in complexity based on different criteria for updating and computing the optimum weights. Adaptive beamforming is known to have resolution and interference rejection capability when the array steering vector is precisely known, however the performance of adaptive beamforming techniques may degrade severely in the presence of mismatches between assumed array response and true array response.

\section{Smart Antenna}

Smart antennas also known as adaptive array antennas, multiple antennas and recently MIMO are antenna arrays with smart signal processing algorithms used to identify spatial signal signature such as the direction of arrival (DOA) of the signal, and use it to calculate beamforming vectors, to track and locate the antenna beam on the mobile/target. The antenna could optionally be any sensor.

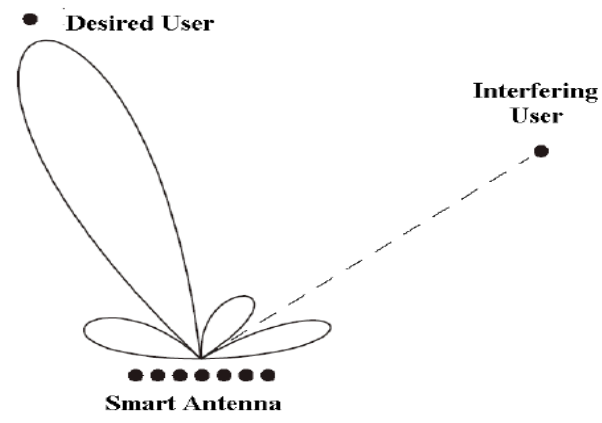

Figure: Radiation pattern of smart antenna

A smart antenna is commonly defined as a multi-element antenna where the signals received at each element are intelligently and adaptively combined to improve the overall performance of the wireless system, with the reverse performed on transmit. Smart antennas have the property of spatial filtering, which makes it possible to receive energy from a particular direction while simultaneously blocking it from another direction. The benefit of smart antennas is that they can increase range and capacity of systems while helping to eliminate both interference and fading. Smart antennas can provide higher system capacities, increase signal to noise ratio, reduce multipath and co-channel interference by steering the main beam towards the user and at the same time forming nulls in the directions of the interfering signal.

The smart antenna system can be divided mainly into three parts.

$>$ The first one performs the direction of arrival (DOA) estimation and determines the number of incoming signals.

$>$ The second part performs the DOA classification. It finds out which signals originate from the user and which ones from the interferers.

$>$ The third part consists in the beamforming algorithm It forms an antenna pattern with a main beam 
steered in the direction of the user, while minimizing the influence of the interfering signals and the noise.

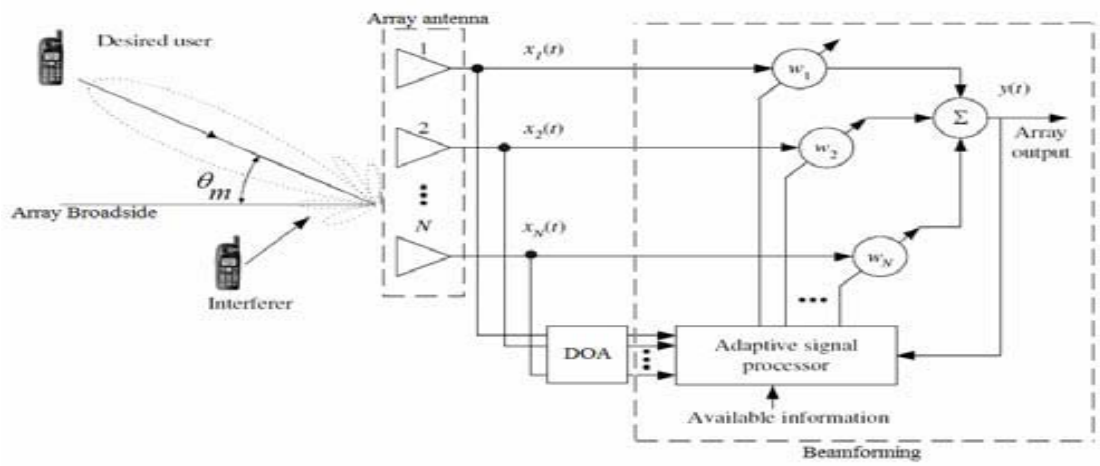

Figure: Functional block diagram of smart antenna system

\subsection{Types of Smart Antenna:}

Smart antennas can be examined in two basic types:-

$>$ Switched beam-A finite number of fixed, predefined patterns or combining strategies (sectors)

$>$ Adaptive array- An infinite number of patterns (scenario-based) that are adjusted in real time.

\subsubsection{Switched Beam Antenna:}

The simplest implementation of a smart antenna is the switched beam system, in which a single transceiver is connected to the RF beamforming unit. The RF beamforming unit, switches to one of the predefined set of beams, according to the received signal power or minimum bit error ratio. The maxima and nulls of the radiation pattern cannot be adjusted for the directions of users, so this technique has limited capabilities as a smart antenna.

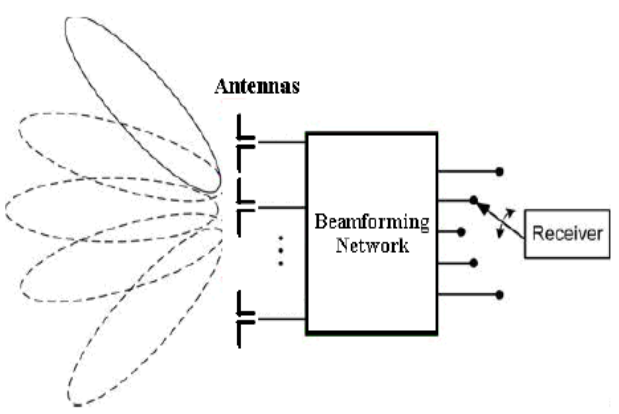

Figure: Switched Beam Antenna

\subsubsection{Adaptive Beamforming Antenna:}

The adaptive beamforming antennas use antenna arrays which are equipped with strong signal processing capability hardware to automatically change the beam pattern in accordance with the changing signal environment. It not only directs maximum radiation in the direction of the desired mobile user, but also introduces nulls at the interfering directions, and tracks the desired mobile user at the same time 6 presents the receiver block of an adaptive beamforming antenna. The adaptation computed is achieved by multiplying the incoming signal with complex weights and then summing them together to obtain the desired radiation pattern. These weights are adaptively by the signal processor to adapt the pattern to the changes in the signal environment. The complex weight computation based on different criteria is incorporated in the signal processor in the form of software algorithms. The transmitter part of a smart antenna has a similar structure with the receiver part. 


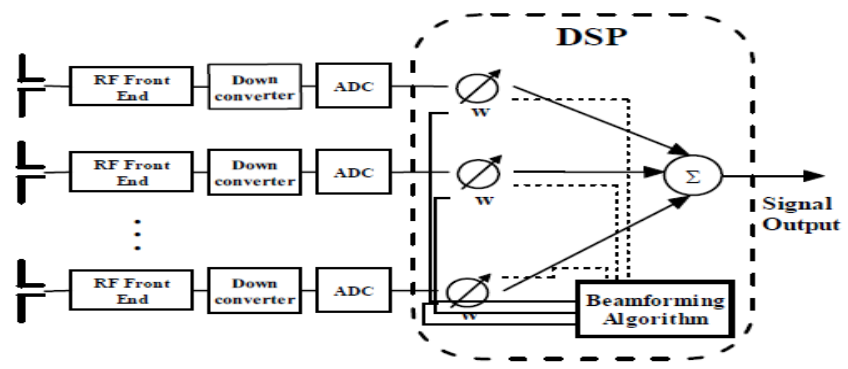

Figure: Receiver block of Adaptive Beam former

The block diagram of the transmitter part is given in Figure 3.7. Here the weights derived from the received signals are also used for the transmitter block. If the uplink and downlink frequencies are close enough, same weights will be adequate for both

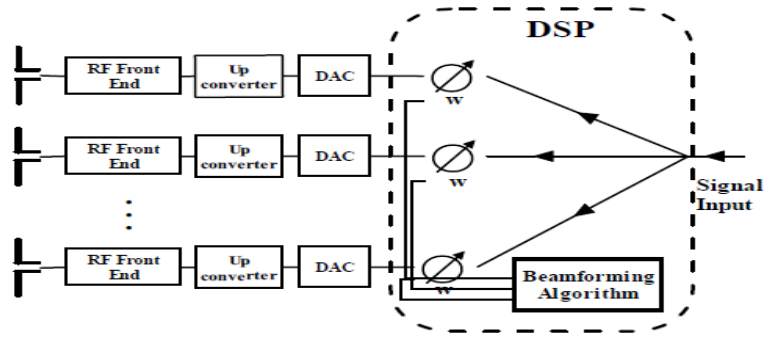

Figure: Transmitter block of Adaptive Beamformer

\section{Smart Antenna System}

There are two types of Smart antenna systems

i) Switched Beam System

ii) Adaptive Antenna System

\section{1 switched beam system}

In terms of radiation patterns, switched beam is an extension of the current microcellular or cellular sectorization method of splitting a typical cell. The switched beam approach further subdivides macro sectors into several micro sectors as a means of improving range and capacity. Each micro sector contains a predetermined fixed beam pattern with the greatest sensitivity located in the center of the beam and less sensitivity elsewhere. The design of such systems involves high-gain, narrow azimuthally beam width antenna elements. The switched beam system selects one of several predetermined fixed-beam patterns (based on weighted combinations of antenna outputs) with the greatest output power in the remote user's channel. These choices are driven by RF or base band DSP hardware and software. The system switches its beam in different directions throughout space by changing the phase differences of the signals used to feed the antenna elements or received from them. When the mobile user enters a particular macro sector, the switched beam system selects the micro sector containing the strongest signal. Throughout the call, the system monitors signal strength and switches to other fixed micro sectors as required. Smart antenna systems communicate directionally by forming specific antenna beam patterns. When a smart antenna directs its main lobe with enhanced gain in the direction of the user, it naturally forms side lobes and nulls or areas of medium and minimal gain respectively in directions away from the main lobe. Different switched beam and adaptive smart antenna systems control the lobes and the nulls with varying degrees of accuracy and flexibility.

\subsection{Adaptive Antenna System}

The adaptive antenna systems approach communication between a user and base station in a different way, in effect adding a dimension of space. By adjusting to an RF environment as it changes (or the spatial origin of signals), adaptive antenna technology can dynamically alter the signal patterns to near infinity to optimize the performance of the wireless system. Adaptive arrays utilize sophisticated signal-processing algorithms to continuously distinguish between desired signals, multipath, and interfering signals as well as 
calculate their directions of arrival. This approach continuously updates it's transmit strategy based on changes in both the desired and interfering signal locations. The ability to track users smoothly with main lobes and interferers with nulls ensures that the link budget is constantly maximized because there are neither micro sectors nor predefined patterns. Both types of smart antenna systems provide significant gains over conventional sectored systems. The low level of interference on the left represents a new wireless system with lower penetration levels. The significant level of interference on the right represents either a wireless system with more users or one using more aggressive frequency reuse patterns. In this scenario, the interference rejection capability of the adaptive system provides significantly more coverage than either the conventional or switched beam system.

\section{Objective Of Smart Antenna System}

The dual purpose of a smart antenna system is to augment the signal quality of the radio-based system through more focused transmission of radio signals while enhancing capacity through increased frequency reuse.

\begin{tabular}{|c|c|}
\hline Feature & Benefit \\
\hline $\begin{array}{l}\text { Signal Gain-Inputs } \\
\text { from multiple antennas } \\
\text { are combined to } \\
\text { optimize available power } \\
\text { Required to establish given level of coverage. }\end{array}$ & $\begin{array}{l}\text { Better Range / Coverage-- } \\
\text { Focusing the energy sent out into } \\
\text { the cell increases base station } \\
\text { Range and coverage. Lower power } \\
\text { requirements also enable a greater } \\
\text { battery life and smaller/lighter } \\
\text { Handset size. }\end{array}$ \\
\hline $\begin{array}{l}\text { Interference Rejection } \\
\text {-Antenna pattern can } \\
\text { be generated toward co } \\
\text { channel interference } \\
\text { sources, improving the } \\
\text { signal-to-interference } \\
\text { ratio of the received } \\
\text { signals. }\end{array}$ & $\begin{array}{l}\text { Increased Capacity-Precise } \\
\text { control of signal nulls quality } \\
\text { and mitigation of interference } \\
\text { combine to frequency reuse } \\
\text { reduce distance (or cluster size), } \\
\text { improving capacity. Certain } \\
\text { adaptive technologies (such as } \\
\text { space division multiple access) } \\
\text { support the reuse of frequencies } \\
\text { within the same cell. }\end{array}$ \\
\hline $\begin{array}{l}\text { Spatial Diversity- } \\
\text { Composite information } \\
\text { from the array is used } \\
\text { to minimize fading } \\
\text { and other undesirable } \\
\text { effects of multipath } \\
\text { Propagation. }\end{array}$ & $\begin{array}{l}\text { Multipath Rejection-can reduce } \\
\text { the effective delay spread of the } \\
\text { channel, allowing higher bit rates } \\
\text { to be supported without the use of } \\
\text { an equalizer, improved bit error } \\
\text { rate (due to decreased amount of } \\
\text { Multipath and ISI). }\end{array}$ \\
\hline
\end{tabular}

\section{Consequences}

The introduction of smart antennas will have a large impact on the performance of cellular networks. It will also affect many aspects of both the planning and deployment of mobile systems. This chapter will discuss the potential benefits and cost factors, and will also briefly describe the implications on radio planning.

\subsection{Capacity Increase}

The principle reason for the growing interest in smart antennas is the capacity increase. In densely populated areas mobile systems are normally interference-limited, meaning that interference from other users is the main source of noise in the system. This means that the signal to interference ratio, SIR, is much larger than the signal to thermal noise-ratio, SNR. Smart antennas will on average, by simultaneously increasing the useful received signal level and lowering the interference level, increase the SIR. Especially, the adaptive array will give a significant improvement. Experimental results report up to $10 \mathrm{~dB}$ increases in average SIR in urban areas.

\subsection{Range Increase}

In rural and sparsely populated areas radio coverage rather than capacity will give the premises for base station deployment. Because smart antennas will be more directive than traditional sector or Omni directional 
antennas, a range increase potential is available. This means that base $\mathrm{s}$ tat ions can be placed further apart, potentially leading to a more cost-efficient deployment. The antenna gain compared to a single element antenna can be increased by an amount equal to the number of array elements, e.g., an eight-element array can provide a gain of eight $(9 \mathrm{~dB})$.

\subsection{New Services}

When using smart antennas the network will have access to spatial information about the users. This information can be used to estimate the positions of the users much more accurately than in existing networks. Positioning can be used in services such as emergency calls and location specific billing. The FCC (Federal that by October 2001 user location information with accuracy of 125 meters RMS error must be provided.

\subsection{Security}

It is more difficult to tap a connection when smart antennas are used. To successfully tap a connection the intruder must be positioned in the same direction as the user as seen from the base station.

\subsection{Reduced Multipath Propagation}

By using a narrow antenna beam at the base station the multipath propagation can be somewhat reduced. The actual reduction depends on the scenario, and is not always significant. Although channel equalizers and RAKE receivers most often will handle and even exploit the multipath components, on very high-speed connections this may not be the case. Potentially, the reduction of multipath propagation can be used to ease the requirement on future modem design.

\section{Conclusion}

The main goal of this article has been to provide an overview of smart antenna technologies with respect to third-generation mobile communication systems. Obviously that smart antennas at the base stations will be an important technology to provide the necessary capacity and coverage. It also helps to realize new services, e.g., based on user location. From a technology point of view, smart antennas can be seen as an extension of the "conventional" resource allocation schemes used in radio communications. In addition to dividing the space into cells, it will now also be possible to employ space division inside each cell. Different degrees of utilization of the spatial dimension are possible, and different steps have been described here. Smart antenna technology is a broad concept and implementations range from simple techniques that involve switching between lobes to advanced algorithms maximizing the received signal to interference ratio. Implementation of smart antennas is done using array antennas. The techniques for Beamforming with array antennas are well known, and must be employed in both duplex directions for the improvements to be substantial. However, with rapid channel variations it is not a trivial task to provide optimum beam forming, especially for the downlink direction. The use of smart antennas is not purely a radio transmission issue. It also influences network services such as handover and connection setup. Introducing the spatial domain in the resource management system makes this more complex.

\section{Acknowledgements}

The authors wish to thanks their parents for supporting and Motivating for this work are very thankful to the RKDF IST Bhopal (MP) India for supporting in contribution towards development of this Article.

\section{References}

[1] P. H. Lehne, O. Rostbakken, M. Petersen,"Estimating Smart Antenna Performance from Directional Radio Channel Measurements", Proc. 50th IEEE Vehic. Tech. Conf. - VTC '99 - F a 11, Amsterdam, the Netherlands, Sept. 19-22, 1999, pp. 5761.

[2] G. Tsoulos, M. Beach, J. McGeehan,"Wireless Personal Communications for the 21st Century: European Technological Advances in Adaptive Antennas", IEEE Communications Magazine, September 1997.

[3] Agius A A. Leach S M. Suvannapattana P, Lund T, Saunders S R,"Intelligent Handheld Antennas for Mobile Communications Beyond the 2nd Generation", version 2.0.2, pp. 12.

[4] Trent K,(2001),"Are Smart Antennas the way to Non- Line-of-Sight?", [Online] Available: http://www. shorecliffcommunications.com/magazine/volume.asp. 
[5] Litwa J,"Digital Beam forming in wireless communi-cations, 1996.

[6] Steyskal H,"Digital Beam forming antennas", an introduction, Microwave J, 30, 1987, pp. 107-124.

[7] Martin Cooper, Marc Goldburg,"Intelligent Antennas: Spatial Division Multiple Access", Annual Review of Communications, 1996.

[8] Scot D. Gordon, Martin J. Feuerstein, Michael A. Zhao "Methods for Measuring and Optimizing Capacity in CDMA Networks Using Smart Antennas", Metawave Communications, pp. 103-112, 425-702-5884.

[9] Michael Tangemann, Rupert Rheinschmitt,"Comparison of Upgrade Techniques for Mobile Communication Systems”, IEEE International Conference, pp. 201-205, Vol. 1, 1-5 May 1994.

[10] B. Ludden et al.,"Mobile Subscriber Location using Handset- Assisted Positioning Techniques", $3{ }^{\text {rd }}$ European Personal mobile Communications Conference - EPMCC '99, Paris, France, Mar. 9-11, 1999, pp.6772.

[11] M. Cooper, M. Goldburg,"Intellingent Antennas: Spatial Division Multiple Access", Wireless Annual Review of Communications, 1996. ArrayComm Inc.

[12] P. E. Mogensen, P. Leth-Espensen, "Performance of Beam Steering in a 1/3 Reuse FH-GSM Network",TSUNAMI II Final Wksp., Aalborg, Denmark, Sept. 1998.

[13] R. Bormann et al.,"Functional Tests of Adaptive Antenna Base Stations for GSM", 3rd European Personal mobile Communications Conference - EPMCC '99, Paris, France, Mar. 9-11, 1999, pp. 323-327.

[14] D. Greenaway et al.,"Final Report - TSUNAMI (II), CEC deliverable AC020/ERA/A52/DS/P/155/b1, Aug. 1998.

[15] A. Kuchar, M. Taferner, E. Bonek, “A Run-Time Optimized Adaptive Antenna Array Processor for GSM”, 3rd Euro - pean Personal mobile Communications Conference - EPMCC '99, Paris, France, Mar. 9-11, 1999, pp. $307-312$.

[16] Ch. Santhi Rani, Dr. P V Subbaiah, Dr. K Chennakesava reddy, "Smart Antenna Algorithms for WCDMA Mobile Communication Systems", IJCSNS International Journal of Computer Science and Network Security, Vol. 8, No. 7, July 2008.

[17] Murali Kiruba,"Smart Antennas for Wireless Mobile Communication”, IT toolbox Wireless- 15592, 22 Oct 2004.

[18] An Introduction to Analog and Digital Communications- Simon Haykin, Wiley India Edition, 2007.

[19] Andreas F. Molisch,"Wireless Communications, Wiley India Edition, 2011.

[20] Frank Gross, - Smart Antenna for Wireless Communicationll, Mcgraw-hill, September 14, 2005.

[21] Carl B. Dietrich, Jr., Warren L. Stutzman, Byung-Ki Kim, and Kai Dietze, "Smart Antennas in Wireless Communications: BaseStation Diversity and Handset Beam forming”, lEEE Antennas and Propagation Magazine, Vol. 42, No. 5, October 2000. 